\title{
Differentiation of Malignant Compared to Non-Malignant Cells by Their Bio-Photon Emissions May Only Require a Specific Filter around $500 \mathrm{~nm}$
}

Nirosha J Murugan, Lukasz M Karbowski, Blake T Dotta, David AE Vares, Kevin S Saroka, Robert M Lafrenie and Michael A Persinger*

Biomolecular Sciences, Human Studies and Behavioural Neuroscience Programs, Laurentian University, Ontario, Canada

\begin{abstract}
Emphasis upon early detection of malignant cellular growths rather than imaging could allow earlier intervention Photon emissions from malignant cells even when they constitute a very small proportion of the normal organ has been shown to require a technical understanding of the spectral power density profiles that can be predicted by Cosic's Molecular Resonance Recognition equation. Here we demonstrate experimentally a simpler more robust detection method involving specific filters of photon emissions from cells in culture. Photons from human pancreatic malignant cancer cells displayed conspicuously suppressed spikes of photons within a narrow band (500 $\mathrm{nm})$ but not at $370 \mathrm{~nm}, 420 \mathrm{~nm}, 620 \mathrm{~nm}, 790 \mathrm{~nm}$, or $950 \mathrm{~nm}$ increments compared to non-malignant human embryonic kidney cells. Given the recent demonstration that malignant cells can "store" photons within a specific wavelength when pulsed at the same pattern as a yoked magnetic field and re-emit the photons in this wavelength tens of minutes later, diminishment of power within specific $10 \mathrm{~nm}$ increments of visible wavelength spectra may serve as an early detection of imminent malignancy.
\end{abstract}

Keywords: Photon emissions; $500 \mathrm{~nm}$ suppression; Early detection; AsPc1 cells; HEK293T cells

\section{Short Communication}

Differentiating malignant from nonmalignant cells by methods that are reliable, simple, and quick is a primary goal of cancer science and therapy. Although metabolites or correlates of aberrant chemical pathways are the standard method of estimating detection at present, there is clear evidence that biophoton emissions differ between cells that are considered malignant compared to non-malignant. Those photons may be a primary means of communications between cells and microorganisms has been hypothesized by Trushin [1] and Fels [2]. The biophysical properties of tumors exposed to treatment-related magnetic fields that facilitate photon emissions have been pursued by Saroka et al. [3].

Dotta et al. [4] were the first to demonstrate that cancer cells shift the dominant wavelength of their photon emissions during the several hours after they are removed from standard incubation as they habituate to ambient temperature. The shift across the near-ultraviolet through the visible band into the near-infrared was consistent with the real-time values for cellular activation and proliferation. The direct coupling of the specific wavelength of photon emissions with specific chemical components of cellular pathways was based upon Cosic's [5] calculations of Molecular Resonance Recognition. These mechanisms [6] have resulted in a shift in scientific paradigm to access the latent cancer potentials within cells, organs, and organisms.

Employing wide (visible) range photomultiplier units we have shown that a variety of different human and animal malignant cells exhibit similar spectral power densities that are not displayed by reference or non-malignant cells. Similar differential profiles of spectral power densities have been measured for mice with and without melanoma tumors. Similar differentiation may be possible with arrays of orthogonally placed digital photomultiplier units surrounding a human subject while he or she is sitting in a small, hyperdark $\left(10^{-12}\right.$ $\mathrm{W} \cdot \mathrm{m}^{-2}$ ) room.

In one case of suspected pancreatic neoplasm in a late middleaged human volunteer the spectral profile of the whole body photon emissions while the person was sitting in a hyper-dark environment for only two minutes differed from the reference population but did not differ from the spectral power densities of photons emitted from known malignant pancreatic cancer cells in vitro. The profile was different from malignant cells lines from other organ systems (Figure 1).

In order to discern if simpler methods could be developed to differentiate malignant and non-malignant cells in vitro we measured raw photon counts from digital photomultiplier units whose sensors were placed over the top a plate of cells while maintained in standard incubators [7]. The measurement duration was $22.5 \mathrm{hr}$ which is the range of the software for the photomultiplier units when sampled once every $2.5 \mathrm{~s}$. We employed standard filters for the following wavelengths $370 \mathrm{~nm}, 420 \mathrm{~nm}, 500 \mathrm{~nm}, 620 \mathrm{~nm}, 790 \mathrm{~nm}, 950 \mathrm{~nm}$ [4]. The "bandwidth" for these filters is about $10 \mathrm{~nm}$. Before a result was considered to be robust it was required to be similar with triplicate replications. As shown in Figure 1 we have found that the $500 \mathrm{~nm}$ filter reliably differentiates healthy cells from malignant cells. A similar effect was noted for MDAMB231 (mammary adenocarcinoma cell line). It may be relevant that the application of light at very low intensities near this wavelength produces a maximum suppression of circulating melatonin levels, a natural oncostatic serotonin derivative [8].

The results are consistent with our [9] hypothesis that the photon emissions and ultimately effective treatments are coupled to the Nernst-level values of the plasma membrane of cancer cells compared to non-malignant cells.

${ }^{*}$ Corresponding author: Persinger MA, Biomolecular Sciences, Human Studies and Behavioural Neuroscience Programs, Laurentian University, Sudbury, Ontario P3E 2C6, Canada, Tel: +017056754824; E-mail: mpersinger@lauentian.ca, drpersinger@neurocog.ca

Received December 18, 2015; Accepted June 27, 2016; Published June 29, 2016

Citation: Murugan NJ, Karbowski LM, Dotta BT, Vares DA, Saroka KS, et al (2016) Differentiation of Malignant Compared to Non-Malignant Cells by Their BioPhoton Emissions May Only Require a Specific Filter around $500 \mathrm{~nm}$. J Cancer Sci Ther 8: 168-169. doi:10.4172/1948-5956.1000409

Copyright: @ 2016 Murugan NJ, et al. This is an open-access article distributed under the terms of the Creative Commons Attribution License, which permits unrestricted use, distribution, and reproduction in any medium, provided the original author and source are credited. 
Citation: Murugan NJ, Karbowski LM, Dotta BT, Vares DA, Saroka KS, et al. (2016) Differentiation of Malignant Compared to Non-Malignant Cells by Their Bio-Photon Emissions May Only Require a Specific Filter around 500 nm. J Cancer Sci Ther 8: 170-171. doi:10.4172/19485956.1000409

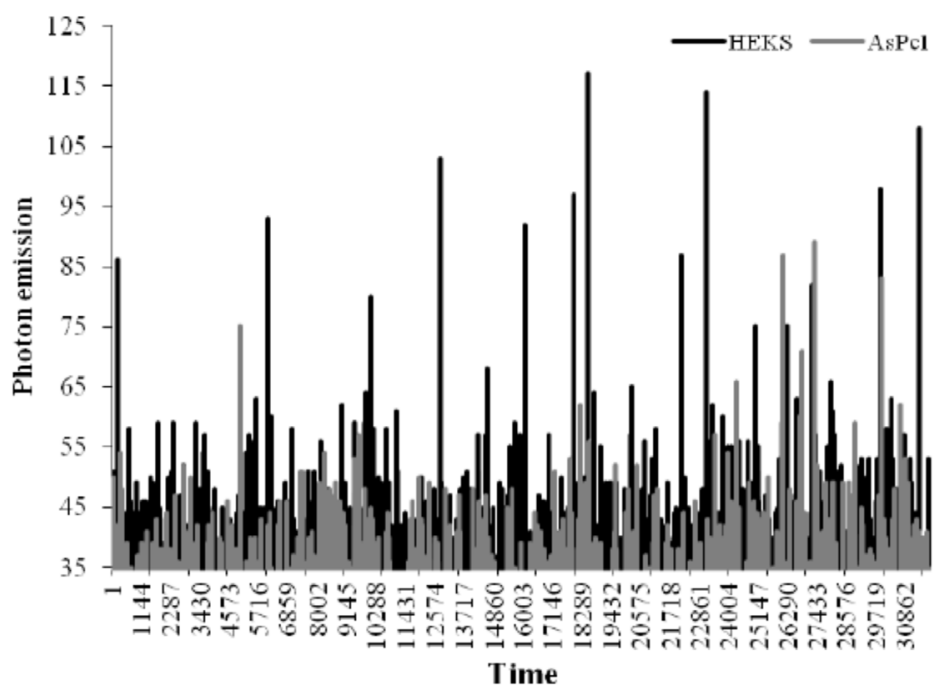

Figure 1: Numbers of photons per s over $22.5 \mathrm{hr}$ from AsPc1 (human pancreatic malignant cancer cells, grey) compared to non-malignant HEK293T (human embryonic kidney cell, black) through the $500 \mathrm{~nm}( \pm 10 \mathrm{~nm})$ filter of digital photomultiplier unit. Note the attenuation of normal spikes of photon emissions in the pancreatic cancer cells.

Karbowski et al. [10] recently demonstrated for the first time that specific photon energies (wavelengths) are "stored" within cultures of malignant cells for at least an hour when the LED-generated light is pulsed concurrently with a specific weak temporally patterned magnetic field. The "re-emitted" photons were the same wavelength as those that had been previously applied. Salari et al. [11] have just demonstrated that the spontaneous cellular ultraweak photon emissions are the most likely source of the "dark noise" from retinal cells which re-affirms the ubiquitous presence $[1,2]$ of biophotons.

Calculations by Persinger [12] extended the inferences by Salari et al. [11] and strongly suggest that spontaneous photon emissions from cells are fractional "re-emissions" that originate from quantum sources [9]. They reflect properties of the upper boundary of the rest mass of photons and the strong possibility that the total biomass on the planet is the energy equivalent of the cumulative incident solar energy since abiogenesis. If the effect we measured in the present experiment generalizes to the whole organism perhaps an inexpensive technique with specific geometry and filters for early screening could be developed.

\section{Refrences}

1. Trushin MV (2004) Light-mediated "conversation" among microorganisms. Microbiol Res 159: 1-10.

2. Fels D (2009) Cellular communication through light. PLoS One 4: e5086.

3. Saroka KS, Karbowski LM, Murugan NJ, Persinger MA (2015) Evidence of "trapped" voltage spectrum residuals within mouse melanoma tumors for about 30 minutes following brief exposure to treatment-related physiologicallypatterned magnetic fields. Archiv Cancer Res 3: 1-5.
4. Dotta BT, Murugan NJ, Karbowski LM, Lafrenie RM, Persinger MA (2014) Shifting wavelengths of ultraweak photon emissions from dying melanoma cells: their chemical enhancement and blocking are predicted by Cosic's theory of resonant recognition model for macromolecules. Naturwissenschaften 101 $87-94$

5. Cosic I (1994) Macromolecular bioactivity: is it resonant interaction between macromolecules?--Theory and applications. IEEE Trans Biomed Eng 41: 11011114.

6. Karbowski LM, Murugan NJ, Persinger MA (2015) Novel Cosic resonance (standing wave) solutions for components of the JAK-STAT cellular signaling pathway: A convergence of spectral density profiles. FEBS Open Bio 5: 245250.

7. Karbowski LM, Murugan NJ, Dotta BT, Persinger MA (2015) Only $1 \%$ melanoma proportion in non-malignant cells exacerbates photon emissions: implications for tumor growth and metastases. Int J Can Res Molec Mech 1(2): 1-3.

8. Horne JA, Donlon J, Arendt J (1991) Green light attenuates melatonin output and sleepiness during sleep deprivation. Sleep 14: 233-240.

9. Persinger MA, Lafrenie RM (2014) The cancer cell plasma membrane potentials as energetic equivalents to astrophysical properties. Int Lett Chem Phys Astron 17: 67-77.

10. Karbowski LM, Murugan NJ, Persinger MA (2016) Experimental evidence that specific photon energies are "stored" in malignant cells for an hour: the synergism of weak magnetic field-LED wavelength pulses. Biology and Medicine 8: 1 BM-162-16.

11. Salari V, Scholkmann F, Bokkon I, Shahbazi F, Tuszynski J (2016) The Physical Mechanism for Retinal Discrete Dark Noise: Thermal Activation or Cellular Ultraweak Photon Emission? PLoS One 11: e0148336.

12. Persinger MA (2016) Spontaneous photon emissions in photoreceptors: potential convergence of Arrhenius reactions and the latency for rest mass photons to accelerate to Planck unit energies. J Advances Physics 11: 3529 3534. 